\title{
Effect of PAD4 on Nasopharyngeal Carcinoma and Nasopharyngeal Carcinoma Cells
}

\author{
XIANGPING WANG, CHANGJIE HUANG* AND H. CHEN \\ Department of Oncology, The Third Affiliated Hospital of Guangxi Medical University, 13 Dancun Road, Nanning, Guangxi \\ 530031, China
}

\section{Wang et al.: Effect of PAD4 on nasopharyngeal carcinoma}

\begin{abstract}
The purpose of this study was to evaluate the expression of PAD4 in nasopharyngeal carcinoma and to explore the relationship between PAD4 and the basic characteristics of 5-8F cells. Immunohistochemistry was used to evaluate the overexpression of the PAD4 protein in the nasopharyngeal carcinoma tissue of 30 patients diagnosed by biopsy in the Third Affiliated Hospital of Guangxi Medical University from January 2018 to June 2019. At the same time, cell function in vitro was evaluated in 5-8F cell line. Cell proliferation, apoptosis and cell cycle were analysed using the MTT assay and flow cytometry. Reverse transcription quantitative polymerase chain reaction was used to evaluate the ability of invasion and angiogenesis. The expression level of PAD4 was related to the size and stage of the primary tumour, lymph node status and pathological grade. Compared to the control group, the activity of PAD4 knockdown cells decreased significantly, the number of the apoptotic cells and the percentage of cells in G0/G1 phase in the PAD4 siRNA group were significantly higher than that in the control group. The invasion and angiogenic ability of 5-8F cells in PAD4 siRNA group were significantly lower than that in the control group. PAD4 was overexpressed in nasopharyngeal carcinoma, which was related to its poor clinicopathological features. At the same time, silencing PAD4 can inhibit the proliferation and activity of 5-8F cell lines, which may provide a potential therapeutic target for patients with nasopharyngeal carcinoma.
\end{abstract}

Key words: Nasopharyngeal carcinoma, PAD4, clinical prognosis, cell experiment

Diabetic nasopharyngeal carcinoma (NPC) is a malignant tumour, which is the most common head and neck cancer in southern China ${ }^{[1-2]}$. The pathogenesis of NPC was complex, including Epstein-Barr virus (EBV) infection, exposure to carcinogens and individual susceptibility ${ }^{[1-3]}$. Clinically, NPC showed low differentiation and a high metastasis rate ${ }^{[4-6]}$. Although patients were treated with a combination of chemotherapy and radiotherapy, they still have local recurrence and distant metastasis ${ }^{[5-7]}$. Therefore, it is important to understand the molecular mechanism of NPC. PAD4 was a member of the peptidylarginine deiminase family, which can convert the arginine residues to citrulline in the presence of calcium ions to catalyse the post-translation deamination of proteins. PAD4 plays a role in the development of granulocytes and macrophages, and mediating inflammation and immune responses. PAD4 was often used as a signal protein, arginine deiminase and inflammation-related research. Studies found that PAD4 was overexpressed in many types of cancers, including breast, liver, kidney, bladder, and lung ${ }^{[8]}$. A study found that the expression of PAD4 mRNA was significantly higher in breast fibroadenomas and thyroid adenomas ${ }^{[9]}$. In addition, a study showed that PAD4 was highly expressed in ovarian adenocarcinoma, which was confirmed by reverse transcription quantitative polymerase chain reaction (RT-qPCR) and Western blot analysis ${ }^{[10]}$. It has been reported that the PAD4 may be related to the increase of citrullinated antithrombin in serum samples of patients with colorectal adenocarcinoma ${ }^{[11]}$. Thus, the detection of PAD4 gene abnormalities can provide new ideas for the early diagnosis and clinical intervention of various cancers. To study the function of PAD4 in the prognosis of NPC and 5-8F NPC cells, the expression of PAD4 was detected in 30 human NPC tumour samples, the prognostic value of PAD4 in patients with NPC as well as the biological function of PAD4 in 5-8F NPC cell line was evaluated. From January 2018 to June 2019, 30 patients comprising of 17 males and 13 females in the age range of 25-78 y (median age $44 \mathrm{y}$ ) with NPC diagnosed by biopsy in the Third Affiliated Hospital of Guangxi Medical University were included in the study. All specimens were confirmed by pathology and classified according to World Health Organization (WHO) tumour metastasis staging system. The patients with a history of radiotherapy or chemotherapy were excluded, and all patients signed a written informed 
consent form. According to the manufacturer's instructions, the EnVision detection system was used for the immunohistochemical staining of NPC tissue sections. In short, the NPC tissue was fixed in $4 \%$ paraformaldehyde for $24 \mathrm{~h}$ and embedded in paraffin to make sections and 6 consecutive sections $(4 \mu \mathrm{m})$ were obtained from each tissue. The sections were incubated with PAD4 first antibody (1:500) at $4^{\circ}$ overnight. Then, the sections were treated with HRP-labelled secondary antibodies (1:1000). The negative control was the sections without incubation with the first antibody. Percent positive staining cells was independently evaluated by 3 pathologists $(0: 0 \%, 1: 1-10 \%, 2$ : 11-60 \%, 3: $61-75 \%$ and 4: 76-100\%), the average staining intensity was calculated, the average score $<3$ was regarded as the weak PAD4 protein expression and the average score $\geq 3$ was regarded as strong. Human NPC cell line 5-8F was purchased from the China Centre for Type Culture Collection (Wuhan) and cultured in RPMI-1640 medium supplemented with 10 $\%$ fetal bovine serum (FBS), $100 \mu \mathrm{g} / \mathrm{ml}$ penicillin and $100 \mu \mathrm{g} / \mathrm{ml}$ streptomycin $\left(37^{\circ}, 5 \% \mathrm{CO}_{2}\right)$. For RNA interference, according to the manufacturer's instructions, 5-8F cells were transfected with $50 \mathrm{nM}$ PAD4 small interfering RNA (5'-GGCAAAGAGAUUGACCUGG-3', Thermo Fisher Scientific) and Lipofectamine 2000 (Thermo Fisher Scientific). The scramble siRNA and lipofectamine simulation treatment were used as control. The cells were collected and treated with RIPA buffer (Wuhan Seville Technology Co., Ltd.) on the ice. The extracted protein was quantified by BCA kit (Wuhan Servicebio Technology Co., Ltd.), separated by $10 \%$ SDS-PAGE and then transferred to a PVDF membrane. After being blocked with TBS $(0.1 \%$ Tween- $20+5 \%$ BSA) for $1 \mathrm{~h}$, the membrane was incubated with the antibodies against PAD4 (1VOR $500)$ or $\beta$-actin $(1: 1000)$ overnight at $4^{\circ}$. The membrane was then incubated with HRP-labelled goat antimouse second antibody (1:1000) for $1 \mathrm{~h}$. A fluorescence imaging system was used to analyse the results. The density of the protein bands was analysed by Image $\mathrm{J}$ software. Total RNA of cells was extracted using TRIzol reagent (Thermo Fisher Scientific). According to the manufacturer's instructions, $5 \mu \mathrm{g}$ of total RNA was reverse transcribed into cDNA using PrimeScript ${ }^{\mathrm{TM}} \mathrm{RT}$ kit. The mRNA expression level of a specific gene was detected by SYBR Green kit and evaluated by $2^{-\Delta \Delta} \mathrm{Cq}$ method. The thermal cycle conditions were as follows, $95^{\circ}$ pre-denaturation for $5 \mathrm{~min}, 95^{\circ}$ denaturation for
$30 \mathrm{sec}, 57^{\circ}$ annealing for $30 \mathrm{sec}$ and $4-5$ cycles at $72^{\circ}$ for $1 \mathrm{~min}$. The primers were synthesized by Thermo Fisher Scientific (Table 1). Cells were inoculated in 96well plates at the density of $1 \times 10^{3}$ cells per well and cultured for 24,48 , and $72 \mathrm{~h}$. The cell viability was evaluated by MTT assay. MTT reagent $(5 \mathrm{mg} / \mathrm{ml})$ was added to each well and incubated in the dark for $3 \mathrm{~h}$ $\left(37^{\circ}, 5 \% \mathrm{CO}_{2}\right)$. DMSO $(100 \mu \mathrm{l} /$ well $)$ was added to each well, and the absorbance at $490 \mathrm{~nm}$ was recorded by the enzyme labelling instrument. After the cells were collected $\left(1 \times 10^{6}\right)$, the cell cycle was evaluated using the cell cycle analysis kit and apoptosis was evaluated with the apoptosis detection kit (phycoerythrin Annexin V). After staining, the cells were analysed by the flow cytometry. The invasive ability of $5-8 \mathrm{~F}$ cells was determined by Transwell. The filter was pre-coated with $50 \mu \mathrm{l}$ matrigel, and incubated in a humidified incubator for polymerization. The under well was filled with DMEM medium (10\% FBS). A total of $0.2 \mathrm{ml}$ cells were inoculated into the upper well $\left(2 \times 10^{5}\right.$ cells/ compartment) and incubated at $37^{\circ}$ for $24 \mathrm{~h}$ and the cells on the upper surface of the filter were removed with the cotton swabs. The cells passing through the filter membrane were fixed with $10 \%$ methanol for 5 $\min \left(4^{\circ}\right)$, stained with $0.5 \%$ eosin for $30 \min \left(37^{\circ}\right)$, and counted under light microscope. The data were analysed using Prism statistical software and the measurement data were expressed by mean \pm standard deviation and the data between two groups were analysed by t-test or one-way ANOVA. Each experiment was conducted at least three times. $\mathrm{p}<0.05$ means the difference was statistically significant. Protein expression of PAD4 in the NPC tissue was detected by immunohistochemistry. The results showed that the expression level of PAD4 was not related to the sex and age of the patients. However, the expression level of PAD4 was related to the tumour size, stage, lymph node status, and pathological grade. The high expression of PAD4 was

TABLE 1: PRIMER SEQUENCES USED IN RT-qPCR

\begin{tabular}{ll}
\hline Gene & \multicolumn{1}{c}{ Sequence } \\
\hline PAD4 & F: AAGGAAAGAGATTGACCTGG \\
& R: GAAGCTACAGTCCGTCGATTC \\
Bcl-2 & F: GGTGGGGTCATGTGTGG \\
& R: CGGTTCAGGTACTCAGTCATCC \\
Bax & F: CCCGAGAGGTCTTTTTCCGAG \\
& R: CCGAGCCCATGATGGTTCTGAT \\
VEGF & F: AGGGCAGAATCATCACGAAGT \\
& R: AGGGTCTCGATTGGATGGCA \\
GAPDH & F: CTGGGCTACACTGAGCACC \\
& R: AAGTGGTCGTTGAGGGCAATG
\end{tabular}


significantly correlated with T3-T4 $(\mathrm{P}=0.005), \mathrm{N} 2-\mathrm{N} 3$ $(\mathrm{P}=0.037)$, and III-IV ( $\mathrm{P}=0.014)$ (Table 2). To identify the function of PAD4 in NPC, the siRNA knockdown of PAD4 was carried out. The results of Western blotting and RT-qPCR showed that the expression of mRNA and protein of PAD4 in siRNA transfected cells was inhibited compared with the control group and scramble siRNA group ( $\mathrm{p}<0.05$, fig. $1 \mathrm{~A}$ and $1 \mathrm{~B})$. The effect of PAD4 siRNA on the viability of 5-8F cells was detected using the MTT assay. The results showed that compared to the control group, the activity of PAD4 knockdown cells decreased at 48 and 72 h (fig. 2).
In order to evaluate whether the decrease of cell proliferation induced by PAD4 siRNA was related to apoptosis and cell cycle arrest, apoptosis rate and cell cycle of $5-8 \mathrm{~F}$ cells were analysed. The results indicated that the number of apoptotic cells in the PAD4 siRNA group was higher than that in the control group and scramble siRNA group (fig. 3A). Compared to the control group, the mRNA expression level of Bcl-2 decreased and the expression level of Bax increased in the PAD4 siRNA group (fig. 3B). Cell cycle analysis showed that the percentage of cells in the G0/G1 phase increased significantly compared to the control group

TABLE 2: THE RELATIONSHIP BETWEEN PAD4 AND PATHOLOGICAL CHARACTERISTICS OF PATIENTS

\begin{tabular}{|c|c|c|c|c|}
\hline \multirow[b]{2}{*}{ Variable } & \multirow[b]{2}{*}{ Cases, $\mathrm{n}$} & \multicolumn{2}{|c|}{ PAD4 expression (\%) } & \multirow[t]{2}{*}{$p$ value } \\
\hline & & Low expression & High expression & \\
\hline Sex & & & & 0.315 \\
\hline Male & 17 & $7(58.33)$ & $10(55.56)$ & \\
\hline Female & 13 & $5(41.67)$ & $8(44.44)$ & \\
\hline Age $(y)$ & & & & 0.853 \\
\hline$<60$ & 23 & $12(80.00)$ & $11(73.33)$ & \\
\hline$\geq 60$ & 7 & $3(20.00)$ & $4(26.67)$ & \\
\hline Tumor stage & & & & 0.005 \\
\hline $\mathrm{T} 1-\mathrm{T} 2$ & 20 & $15(78.95)$ & $5(45.46)$ & \\
\hline T3-T4 & 10 & $4(21.05)$ & $6(54.54)$ & \\
\hline Lymph node status & & & & 0.003 \\
\hline N0-N1 & 23 & $18(90.00)$ & $5(50.00)$ & \\
\hline N2-N3 & 7 & $2(10.00)$ & $5(50.00)$ & \\
\hline Pathological grading & & & & 0.001 \\
\hline$|-| \mid$ & 24 & $19(95.00)$ & $5(50 \%)$ & \\
\hline III-IV & 6 & $1(5.00)$ & $5(50 \%)$ & \\
\hline Tumor size & & & & 0.003 \\
\hline$<6 \mathrm{~cm}$ & 22 & $16(84.21)$ & $6(54.54)$ & \\
\hline$\geq 6 \mathrm{~cm}$ & 8 & $3(15.79)$ & $5(45.46)$ & \\
\hline
\end{tabular}

A

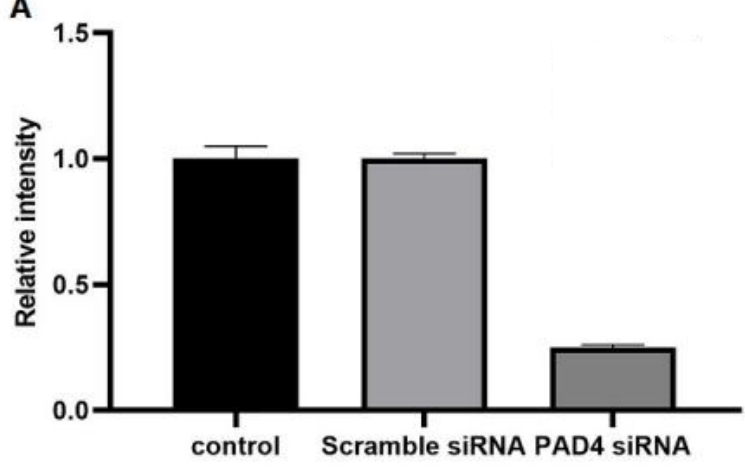

B

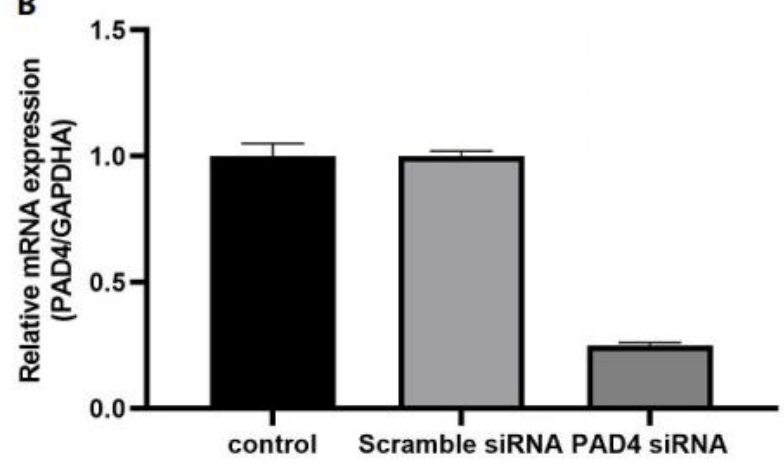

PAD4

$\beta$-actin

Fig. 1: PAD4 siRNA downregulates the expression of PAD4 in 5-8F cells

(A) Western blotting and (B) RT-qPCR were used to evaluate the expression of PAD4, (-) Control, (-) Scramble SiRNA (-) PAD4 siRNA 
and scramble siRNA group, while the percentage of cells in the $S$ phase and G2/M phase decreased significantly in the PAD4 siRNA group (fig. 3C).

Invasive ability of 5-8F cells in the PAD4 siRNA group was evaluated by Transwell. The results showed that invasive ability of the $5-8 \mathrm{~F}$ cells in the PAD4 siRNA group was significantly lower than that in the control group and scramble siRNA group ( $p<0.05$, fig. 4A). In addition, the expression level of the vascular endothelial growth factor (VEGF) in 5-8F cells was

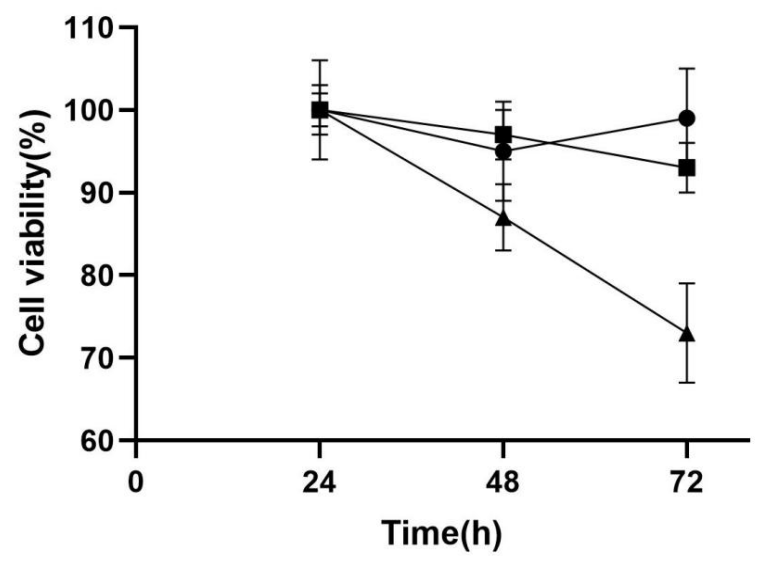

Fig. 2: Effect of KIF2A gene knockout on the proliferation of 5-8F cells

MTT assay was used to detect the effect of KIF2A gene knockout on the proliferation of 5-8F cells, (- - ) Control, (一匹) Scramble siRNA (- - -) PAD4 siRNA evaluated by RT-qPCR. The expression of VEGF in the PAD4 siRNA group was significantly lower than that in the control group and scramble siRNA group $(\mathrm{p}<0.05$, fig. 4B). NPC is a common malignant tumour, originating from the nasopharyngeal epithelium ${ }^{[12-14]}$. The long-term survival rate of the patients with NPC was low. Therefore, the study of the molecular mechanism of the occurrence and development of NPC is of great significance to improve the treatment of NPC. There was the overexpression of PAD4 in many malignant tumors, but this phenomenon was not found in the benign tumors, indicating that the imbalance of PAD4 activity plays an important role in the cancer progression ${ }^{[15]}$. Interestingly, blood PAD4 levels also increased in patients with malignant cancer and then decreased significantly after tumor resection ${ }^{[15]}$. Therefore, the purpose of this study is to evaluate the expression of PAD4 in NPC and to explore the relationship between the expression of PAD4 and the basic characteristics of 5-8F cells. In NPC patients, the expression level of PAD4 was not related to the sex and age of the patients. However, the level of PAD4 was significantly correlated with the tumour stage, lymph node status, and pathological grade, which suggests that the high level of PAD4 may be related to the malignant degree of NPC. Similarly, in previous studies, the mRNA and protein expression levels of PAD4 in the grade III-IV
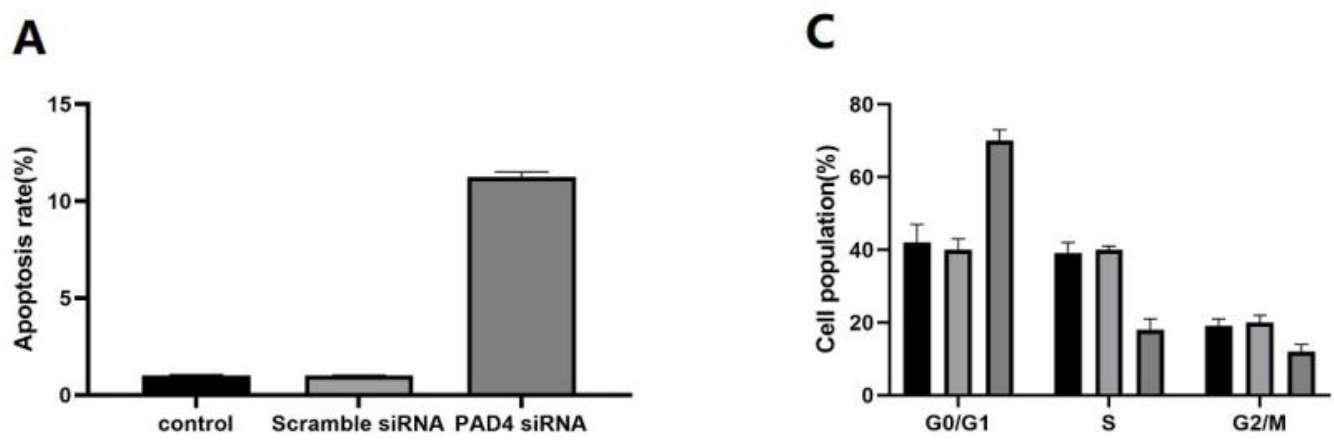

B
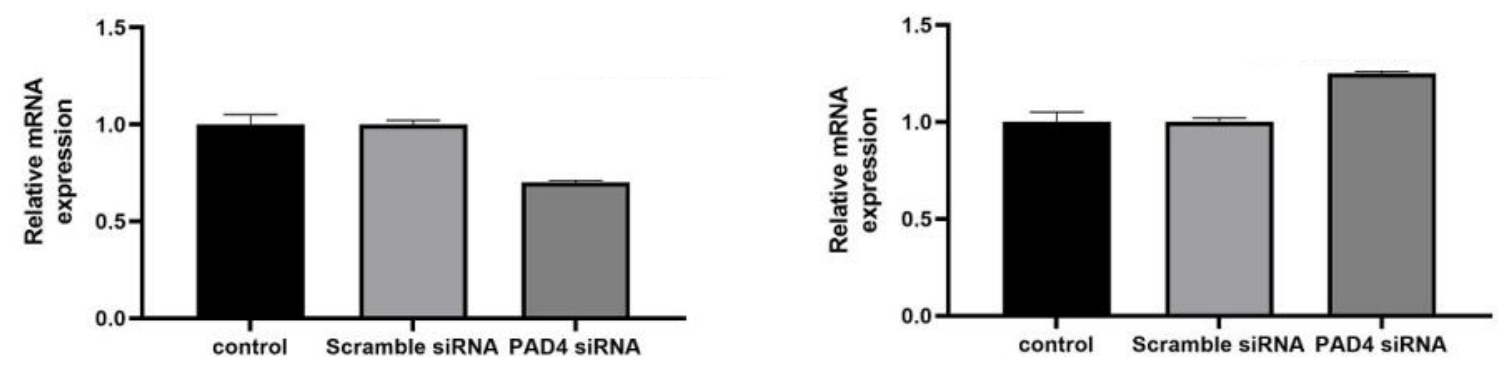

Fig. 3: Effects of PAD4 gene knockout on apoptosis and cell cycle of 5-8F cells

(A) Detection of the apoptotic cells by flow cytometry. (B) Detection of Bcl-2 and Bax expression by RT-qPCR. (C) Detection of cell cycle by the flow cytometry PAD4, $(-)$ Control, $(-)$ Scramble siRNA $(-)$ PAD4 siRNA 
A

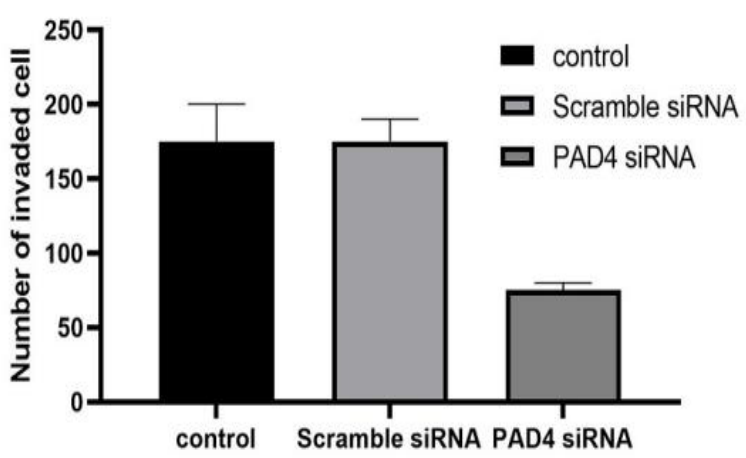

B

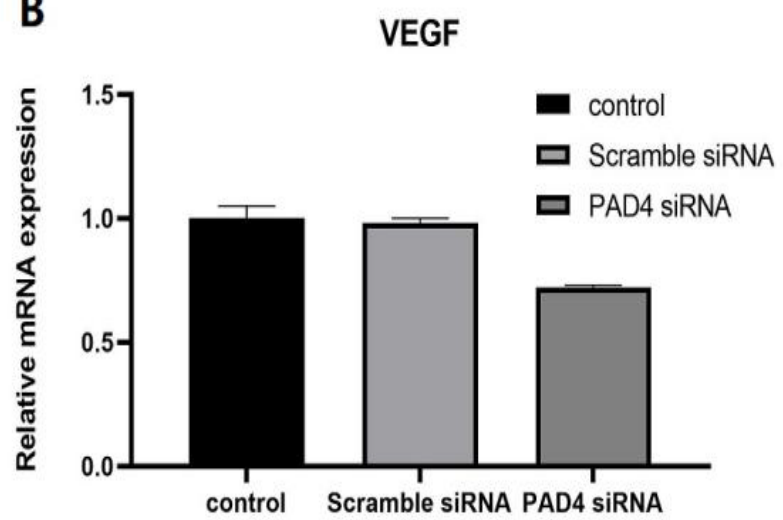

Fig. 4: PAD4 inhibits the invasion and angiogenesis of 5-8F cells in PAD4 siRNA group

(A) Cell infiltration in each group. (B) The expression of VEGF was detected by RT-qPCR.

gastrointestinal cancer tissues were significantly higher than those in the grade I-II and healthy tissues ${ }^{[16]}$. Therefore, the results of this study suggest that PAD4 may be involved in the malignant development of NPC.

Previous studies reported that the PAD4 silencing significantly inhibits the growth and invasion of lung cancer, suggesting that the PAD4 may regulate the viability and invasion of cancer cells ${ }^{[17]}$. In this study, compared with the control group, the proliferation of $5-8 \mathrm{~F}$ cells in the PAD4 siRNA group decreased and the apoptosis increased. The ratio of $\mathrm{Bax} / \mathrm{Bcl}-2$ was an effective method to identify the apoptotic cells. In the PAD4 siRNA group, the mRNA level of the antiapoptotic Bcl-2 decreased, while the mRNA of the pro-apoptotic Bax increased, indicating an increase in the proportion of apoptotic cells ${ }^{[18]}$. The results of the cell cycle showed that the cell number stagnated in the G0/G1 phase of the PAD4 siRNA group were higher than that in the control group. A recent study has shown that the MRE11-RAD50-NBS1 complexes activate PAD4, resulting in spindle turnover and DNA damage ${ }^{[19]}$. Therefore, the PAD4 may be related to the DNA structure and spindle dynamics. In addition, this study showed that compared to the control group, PAD4 siRNA inhibited the invasive ability of the 5-8F cells and the expression of VEGF. Previous studies have reported that the PAD4 was associated with the prognosis of ovarian cancer, and the inhibitory effect of PAD4 can reduce the invasion of ovarian cancer in vitro ${ }^{[20]}$. The downregulation of PAD4 inhibits the invasion of gastric cancer cells by inhibiting the type I membrane matrix metallopeptidase ${ }^{[21]}$. Therefore, PAD4 may be related to the invasion and angiogenesis of $5-8 \mathrm{~F}$ cells. In conclusion, this study showed that the expression of PAD4 was related to the poor clinicopathological features of NPC. In addition, PAD4 may play an important role in the proliferation of NPC and $5-8 \mathrm{~F}$ cells.

\section{Authors contributions:}

Xiangping Wang conceived and designed the experiments; Changjie Huang gave an experimental guidance in the lab; Xiangping Wang, Changjie Huang and Hao Chen performed the experiments; Hao Chen analyzed the data; Hao Chen wrote the paper.

\section{Acknowledgements:}

This work was supported by the Natural Science Foundation of Guangxi Province (Grant No. 2017GXNSFBA198072), the Science Research and Technology Development Key Program of Nanning City (Grant No. 20173018-4), the Nanning Excellent Young Scientist Program and Guangxi Beibu Gulf Economic Zone Major Talent Program (Grant No. RC20190104), the Guangxi Zhuang Autonomous Region Health and Family Planning Commission Self-financing Research Project (Z20180820).

\section{Conflict of interest:}

All authors report no conflicts of interest in this work.

\section{REFERENCES}

1. Wei KR, Zheng RS, Zhang SW, Liang ZH, Li ZM, Chen WQ. Nasopharyngeal carcinoma incidence and mortality in China, 2013. Chin J Cancer 2017;36:90.

2. Tang XR, Li YQ, Liang SB, Jiang W, Liu F, Ge WX, et al. Development and validation of a gene expression-based signature to predict distant metastasis in locoregionally advanced nasopharyngeal carcinoma: A retrospective, multicentre, cohort study. Lancet Oncol 2018;19:382-93.

3. Zhang F, Li J, Xiao H, Zou Y, Liu Y, Huang W. AFAP1-AS1: A novel oncogenic long non-coding RNA in human cancers. Cell Prolif 2018;51:e12397.

4. Tsao SW, Tsang CM, Lo KW. Epstein-Barr virus infection and 
nasopharyngeal carcinoma. Philos Trans R Soc Lond B Biol Sci 2017;372:20160270.

5. He JY, Han P, Zhang Y, Liu YD, Song SJ, Feng GK, et al. Overexpression of Nogo receptor 3 (NgR3) correlates with poor prognosis and contributes to the migration of epithelial cells of nasopharyngeal carcinoma patients. J Mol Med 2018;96:265-79.

6. Zhu JF, Huang W, Yi HM, Xiao T, Li JY, Feng J, et al. Annexin A1-suppressed autophagy promotes nasopharyngeal carcinoma cell invasion and metastasis by PI3K/AKT signalling activation. Cell Death Dis 2018;9:1-6.

7. You R, Cao YS, Huang PY, Chen L, Yang Q, Liu YP, et al. The changing therapeutic role of chemo-radiotherapy for loco-regionally advanced nasopharyngeal carcinoma from two/three-dimensional radiotherapy to intensity-modulated radiotherapy: A network meta-analysis. Theranostics 2017;7:4825-35.

8. Foulquier C, Sebbag M, Clavel C, Chapuy-Regaud S, Al Badine R, Méchin MC, et al. Peptidyl arginine deiminase type 2 (PAD-2) and PAD-4 but not PAD-1, PAD-3, and PAD-6 are expressed in rheumatoid arthritis synovium in close association with tissue inflammation. Arthritis Rheum 2007;56:3541-53.

9. Chang X, Han J, Pang L, Zhao Y, Yang Y, Shen Z. Increased PADI4 expression in blood and tissues of patients with malignant tumors. BMC Cancer 2009;9:40.

10. Brill A, Fuchs TA, Savchenko AS, Thomas GM, Martinod K, De Meyer SF, et al. Neutrophil extracellular traps promote deep vein thrombosis in mice. J Thromb Haemost 2012;10:136-44.

11. Yahagi A, Saika T, Hirano H, Takai-Imamura M, Tsuji F, Aono $\mathrm{H}$, et al. IL-6-PAD4 axis in the earliest phase of arthritis in knock-in gp130F759 mice, a model for rheumatoid arthritis. Rmd Open 2019;5.

12. Xing $\mathrm{H}$, Chen $\mathrm{X}$, Han $\mathrm{Y}$. Role of regenerating gene IA expression on local invasion and survival in nasopharyngeal carcinoma. Biol Res 2017;50:37.

13. Zuo LL, Zhang J, Liu LZ, Zhou Q, Du SJ, Xin SY, et al. Cadherin 6 is activated by Epstein-Barr virus LMP1 to mediate EMT and metastasis as an interplay node of multiple pathways in nasopharyngeal carcinoma. Oncogenesis. 2017;6:1-6.

14. Nakashima K, Hagiwara T, Yamada M. Nuclear localization of peptidylarginine deiminase $\mathrm{V}$ and histone deimination in granulocytes. J Biol Chem 2002; 277:49562-8.

15. Silveira IG, Burlingame RW, von Mühlen CA, Bender AL, Staub HL. Anti-CCP antibodies have more diagnostic impact than rheumatoid factor (RF) in a population tested for RF. Clin Rheumatol 2007;26:1883-9.

16. Suzuki A, Yamada R, Chang X, Tokuhiro S, Sawada T, Suzuki M, et al. Functional haplotypes of PAD4, encoding citrullinating enzyme peptidylarginine deiminase 4, are associated with rheumatoid arthritis. Nat Genet 2003;34:395402.

17. Shi L, Yao H, Liu Z, et al. Endogenous PAD4 in Breast Cancer Cells Mediates Cancer Extracellular Chromatin Network Formation and Promotes Lung Metastasis. Mol Cancer Res 2020;18:735-47.

18. Hong MH, Jie LI, Yan XU. Diagnostic value of anti-Car P antibody and anti-PAD4 antibody for rheumatoid arthritis. Guangdong Med J 2019; 38:2683-9.

19. Fichtner T, Kotarski F, Gärtner U, Conejeros I, Hermosilla C, Wrenzycki $\mathrm{C}$, et al. Bovine sperm samples induce different NET phenotypes in a NADPH oxidase-, PAD4- and Ca++dependent process. Biol Reprod 2020;102:902-14.

20. Bernacki MJ, Czarnocka W, Rusaczonek A, Witoń D, Kęska S, Czyż J, et al. LSD1-, EDS1- and PAD4- dependent conditional correlation among salicylic acid, hydrogen peroxide, water use efficiency and seed yield in Arabidopsis thaliana. Physiol Plantarum 2019;165:369-82.

21. Yadav R, Yoo DG, Kahlenberg JM, Bridges Jr SL, Oni O, Huang $\mathrm{H}$, et al. Systemic levels of anti-PAD4 autoantibodies correlate with airway obstruction in cystic fibrosis. J Cyst Fibros 2019;18:636-45.

This is an open access article distributed under the terms of the Creative Commons Attribution-NonCommercial-ShareAlike 3.0 License, which allows others to remix, tweak, and build upon the work non-commercially, as long as the author is credited and the new creations are licensed under the identical terms

This article was originally published in a special issue, "Biomedical Research in Healthcare Setting" Indian J Pharm Sci 2020:82(3)Spl issue5;66-71 